\title{
Duodenal obstruction induced by retroperitoneal progression of bladder cancer: A report of two cases
}

Koji Tokunaga ${ }^{1}$, Akihiro Furuta ${ }^{1}$, Shigeki Arizono ${ }^{1}$, Yuki Teramoto ${ }^{2}$, Hiromitsu Negoro $^{3}$, Aki Kido ${ }^{1}$, Hiroyoshi Isoda ${ }^{1}$, Kaori Togashi ${ }^{1}$

${ }^{1}$ Department of Diagnostic Imaging and Nuclear Medicine, Kyoto University Graduate School of Medicine, 54 Kawaharacho, Shogoin, Sakyo-ku, Kyoto 606-8507, Japan

${ }^{2}$ Department of Diagnostic Pathology, Kyoto University Graduate School of Medicine, 54 Kawaharacho, Shogoin, Sakyo-ku, Kyoto 606-8507, Japan

${ }^{3}$ Department of Urology, University of Tsukuba Hospital, 2-1-1 Amakubo, Tsukuba, Ibaraki 305-8576, Japan

\section{ORCID}

Koji Tokunaga: 0000-0002-3329-6691

Corresponding author:

Koji Tokunaga

Department of Diagnostic Imaging and Nuclear Medicine, Kyoto University Graduate School of Medicine, 54 Kawaharacho, Shogoin, Sakyo-ku, Kyoto 606-8507, Japan

Phone number: +81-75-751-3760

E-mail address: tokunaga.koji.28m@kyoto-u.jp

Acknowledgment: None

Disclosures: This research did not receive any specific grant from funding agencies in the public, commercial, or not-for-profit sectors.

This is a post-peer-review, pre-copyedit version of an article published in Abdominal Radiology. The final authenticated version is available online at: https://dx.doi.org/10.1007/s00261-018-1874-8. 


\begin{abstract}
Bladder cancer usually forms a papillary structure. Progression along the cavity or membranous structures surrounding the bladder, rectum, or retroperitoneum without formation of a discrete mass is rare. We here present two patients with duodenal obstruction caused by retroperitoneal progression of bladder cancer, in both of whom computed tomography revealed bladder and rectal wall thickening with a malignant target sign, thickened mesorectal fascia with abnormal tissue stranding, and increased perirectal fat density. Both cancers progressed despite treatment, as indicated by faint abnormal tissue stranding and increased retroperitoneal fat density along the retromesenteric plane from the pelvis to the duodenum. Subsequently, both patients developed obstruction in the horizontal portion of the duodenum, still without formation of a mass lesion. These two patients highlight the challenges associated with retroperitoneal invasion by bladder cancer in the absence of a mass lesion and underscore the importance of considering cancer progression in patients with bowel obstruction, even when there is no obvious mass lesion and/or only minor retroperitoneal findings. Progression along the retromesenteric plane may be the key pathway via which progressive bladder cancer results in duodenal obstruction without or with a minor mass lesion.
\end{abstract}

Keywords: Bladder cancer, retroperitoneal progression, duodenal obstruction, retromesenteric plane

\title{
Introduction
}

Bladder cancer, the commonest urinary tract malignancy, usually progresses to form a papillary structure. Progression along the pelvic wall and membranous structures surrounding the bladder, rectum, or retroperitoneum in the absence of a discrete mass is rare. We here present two patients who developed duodenal obstruction as a result of retroperitoneal progression of bladder cancer. 


\section{Patient 1}

The first patient was a 67-year-old man with a 6-month history of dyschezia and more recent dysuria. Initial contrast-enhanced computed tomography (CECT) revealed thickening of the right bladder (Fig. 1) and rectal walls along with a malignant target sign, which comprised alternating layers of hyper-enhancement in the mucosa, submucosa, and adventitia rectalis with hypo-enhancement in the muscular layers [1]. Thickening of the mesorectal fascia with abnormal tissue stranding and increased perirectal fat density were also evident; however, no abnormality was detected in the retroperitoneum or duodenum. Initial pelvic magnetic resonance imaging (MRI) revealed thickening of the rectal wall and mesorectal fascia, with low signal intensity on T2-weighted imaging and high signal intensity on diffusion-weighted imaging (Fig. 2). These findings suggested bladder cancer with invasion of the rectum and mesorectal fascia and obstruction of the rectum.

After conservative treatment of the rectal obstruction, the patient underwent transurethral resection of the bladder tumor (TURBT). Examination of hematoxylin and eosin (HE)-stained sections of the TURBT specimen revealed diffuse cancer cell growth with sporadic muscular invasion (Fig. 3). The clinical stage was cT3aNOM0. The patient subsequently underwent neoadjuvant chemotherapy with gemcitabine/carboplatin followed by total cystectomy; pathological examination of the operative specimen revealed no residual tumor. However, at his 6-month follow-up visit he reported progressive abdominal distension. CECT revealed gastric and duodenal dilation, suggesting duodenal obstruction (Fig. 4). Mild abnormal tissue stranding was also observed in the right retroperitoneum along the retromesenteric plane from the pelvis to the duodenum (Fig. 5). Esophagogastroduodenoscopy revealed no abnormalities in the mucosal layer and endoscopic ultrasonography showed preservation of the layered structure of the duodenal wall with no definite evidence of a mass lesion. An endoscopic biopsy was negative for malignancy. A subsequent laparotomy biopsy of the duodenum and examination of the HE-stained specimen revealed sporadic cancer cells distributed in a semi-linear pattern. These findings were consistent with those of the TURBT specimen and tumor recurrence was diagnosed. 
Examination of E-cadherin immunostained sections showed loss of E-cadherin expression, suggesting epithelial-mesenchymal transition (EMT) of the cancer cells. He died of cancer 1 month after this biopsy.

\section{Patient 2}

The second patient was a 75-year-old man who presented with a 1-month history of difficult and painful urination. A CT revealed a locally advanced, invasive, bladder cancer with rectal stenosis caused by a thickened left bladder wall, thickening of the mesorectal fascia with abnormal tissue stranding, and increased perirectal fat density. No abnormalities were detected in the retroperitoneum or duodenum. Histological confirmation of malignancy was obtained from a TURBT specimen collected prior to treatment. However, cautery-related changes in the specimen obliterated details of the tumor's characteristics (Fig. 6). The clinical stage was cT4bN1M0. A colostomy was constructed to manage the patient's developing rectal obstruction, after which he received chemoradiotherapy for 6 months. He then developed vomiting. CECT revealed an obstruction in the horizontal portion of the duodenum. Additionally, minor abnormal tissue stranding was observed along the retromesenteric plane from the pelvis to the duodenum. Thickening of the bladder wall, mesorectal fascia, and rectal wall were observed; the latter exhibiting alternating layers of hypo- and hyper-enhancement (Fig. 7). Esophagogastroduodenoscopy and endoscopic ultrasonography revealed no abnormalities in the mucosa or other layered structures of the duodenum. In particular, there was no evidence of a definite mass lesion. The patient died of cancer 1 month later.

\section{Discussion}

Bladder cancer typically presents as a superficial tumor localized to the bladder. Approximately $20 \%$ of affected patients present with muscular wall invasion, fewer than $5 \%$ with locally advanced (i.e., clinically extravesical) disease, and approximately 5\% with clinically apparent metastatic disease [2].

In both of the present patients, CT and MRI revealed increased intrapelvic fat density, 
thickening of the bladder and rectal walls and mesorectal fascia with abnormal tissue stranding, and a malignant target sign in the rectum. The latter sign, which denotes cancer infiltration, comprises hyper-enhancement of the mucosa and submucosa (i.e., the inner layer of the intestine), and of the adventitia rectalis, subserosa, and serosa, if applicable (i.e., the outer layer) [1]. With progressive cancer infiltration over time and the eventual development of homogeneous thickening of the intestinal wall, the presence of a malignant target sign can indicate development of ileus [1]. CECT images may also show lesions without definite contiguity; absence of mass formation; and few, minor, or no retroperitoneal abnormalities. These findings are characteristic of tumor spread across the bladder wall to the rectum and are similar to those found in association with other cancers with discohesive growth patterns such as diffuse gastric cancer and lobular breast cancer [3]. They can also occur in rare types of bladder cancer that develop scirrhous-like metastases, including high-grade urothelial cancer with a marked EMT, as was the case with our first patient. EMT is associated with loss of epithelial characteristics and development of motility, invasiveness, and an ability to migrate while retaining expression of cell-adhesion molecules, including E-cadherin $[4,5]$. These characteristics can lead to rapid and wide-spread retroperitoneal progression without formation of a mass. Although detailed histological information was not available in our second patient, the similarities in imaging findings between the patients led us to infer that his tumor was poorly or undifferentiated and had a discohesive growth pattern.

Most duodenal obstruction associated with malignancy results from contiguous spread from cancers of the pancreas or gallbladder. Secondary involvement of the duodenum or adjacent retroperitoneum is common with metastases from lung cancer, renal cell carcinoma, malignant melanoma, and lobular breast cancer; however, development of obstruction is not [6]. Duodenal obstruction is rarely caused by a noncontiguous isolated lesion from bladder cancer, obstructive lesions usually being accompanied by subtle retroperitoneal abnormal tissue stranding, as was the case with both of our patients. In a previous report of bladder cancer with duodenal obstruction, a metastatic infiltrative retroperitoneal soft tissue mass encasing the second/third portion of the duodenum was identified in the absence of any other 
retroperitoneal abnormalities [6], whereas in another report the cancer extended from the bladder to the pancreas via the retroperitoneum, as evidenced by soft tissue stranding in the retroperitoneum [7]. As in the latter report, in both of our patients the initial CT revealed no abnormalities in the retroperitoneum or around the duodenum, whereas subsequent CTs after development of duodenal obstruction showed mild abnormal tissue stranding along the retromesenteric plane from the pelvis to the duodenum. The absence of a definite mass lesion or destruction of the layered structure of the duodenal wall suggested retroperitoneal progression of bladder cancer. The retromesenteric plane surrounds the horizontal portion of the duodenum and pancreas and extends to the pelvic region [8-10]. Once a cancer has infiltrated into and progressed along this plane, it can spread and progress around the horizontal portion of the duodenum, thus causing duodenal obstruction without formation of a mass lesion, especially when EMT has been detected in the primary tumor.

In conclusion, our findings highlight the poor prognosis of patients with high histological grade, high stage, and rapidly progressive non-mass-forming bladder cancer combined with thickening of the mesorectal fascia, abnormal tissue stranding or sheet formation in the pelvis and retromesenteric plane, presence of the malignant target sign in the rectum, and of those with pathologically proven high-grade urothelial cancer with marked evidence of EMT. Such patients appear to be highly prone to non-mass-forming progression in the retroperitoneal space and consequent duodenal obstruction.

\section{Compliance with Ethical Standards}

Conflicts of interest: The authors declare that they have no conflicts of interest.

Ethical approval: All procedures performed in studies involving human participants were in accordance with the ethical standards of the institutional and/or national research committee and with the 1964 Helsinki Declaration and its later amendments or comparable ethical standards.

Informed consent: The requirement for written informed consent was waived for this case report on the basis of the opt-out system of our institution. 


\section{References}

1. Gollub MJ, Schwartz MB, Shia J (2009) Scirrhous metastases to the gastrointestinal tract at CT: the malignant target sign. AJR 192:936-940. https://doi.org/10.2214/AJR.08.1152.

2. Bandireddy M, Baffy N (2017) An uncommon cause of gastric outlet obstruction: a case report. Medicine (Baltimore) 96:e7059. https://doi.org/10.1097/MD.0000000000007059.

3. Nabbout P, Furr J, Paari M, Slobodov G (2012) Plasmacytoid urothelial carcinoma of the bladder metastatic to the stomach: a case report. Case Rep Urol 2012:715951. https://doi.org/10.1155/2012/715951.

4. Yeung KT, Yang J (2017) Epithelial-mesenchymal transition in tumor metastasis. Mol Oncol 11:28-29. https://doi.org/10.1002/1878-0261/12017.

5. Kalluri R, Weinberg RA (2009) The basics of epithelial-mesenchymal transition. J Clin Invest 119: 1420-1428. https://doi.org/10.1172/jci39104.

6. Hawtin K, Kent A, Collins C, Blunt D (2009) Metastatic bladder cancer presenting as duodenal obstruction. Ann Acad Med Singapore 38:914-915.

7. Iqbal MA, Lawatsch EJ, Coyle DJ, Rowe JJ, Li R, Dua KS, Kochar MS (2006) Signet-ring cell carcinoma of the urinary bladder mimicking retroperitoneal fibrosis. Wisconsin Med $\mathbf{J}$ $105 ; 55-58$.

8. van Leeuwen MS and Feldberg MAM. The extraperitoneal spaces: Normal and pathologic anatomy. Compartmentalization of the Anterior Pararenal Space. In Meyers MA, Charnsangavej C, Oliphant M (2011) Mayers' Dynamic radiology of the abdomen, 6th ed. New York, NY. pp 152-158. https://doi.org/10.1007/978-1-4419-5939-3.

9. Takeguchi T, Ibukuro K (2011) Anatomy of the Retroperitoneal Space on CT. Jpn J Diag Imaging 31:1130-1140. (in Japanese, English abstract available)

10. Tirkes T, Sandrasegaran K, Patel AA, Hollar MA, Tejada JG, Tann M, Akisik FM, Lappas JC (2012) Peritoneal and retroperitoneal anatomy and its relevance for cross-sectional imaging. Radiographics 32:437-451. https://doi.org/10.1148/rg.322115032. 


\section{Fig. 3 Pathological findings (Patient 1)}

Photomicrographs of sections from a specimen obtained by transurethral resection of the bladder tumor. (a) Hematoxylin and eosin- (HE) stained low-power field image showing a diffuse growth pattern. (b) HE-stained low-power field image showing some sporadic intramuscular cancer cell invasion. (c) HE-stained high-power field image showing some signet-ring cell formation (arrows). (d) Photomicrographs of sections from a laparotomy biopsy specimen of the duodenum. HE-stained high-power field image showing sporadic distribution of cancer cells in a semi-linear pattern (arrows). (e) E-cadherin immunostained high-power field image showing absence of E-cadherin expression.

Fig. 4 Contrast-enhanced computed tomography (Patient 1) after development of abdominal distention.

Dilation of the stomach and duodenum (a), suggestive of duodenal obstruction in the horizontal portion is visible (b, c, black arrows). Mild abnormal tissue stranding is visible in 
the right retroperitoneum (b-d, black arrow heads).

Fig. 5 Axial contrast-enhanced computed tomography image (Patient 1) after development of abdominal distention.

Actual image (a) and schematic depiction of the same image (b). Minor abnormal tissue stranding (yellow area) without formation of a definite mass can be seen along the retromesenteric plane (blue area). The pink line represents the parietal peritoneum; areas "C" and " $\mathrm{D}$ " are the ascending colon and duodenum, respectively.

Fig. 6 Pathological findings (Patient 2)

Photomicrograph of section from a specimen obtained via transurethral resection of the bladder tumor (HE-stained low-power field image) showing intramuscular invasion by poorly differentiated cancer cells (arrows). Cautery-related changes have obliterated the details.

Fig. 7 Coronal (a) and axial (b-d) contrast-enhanced computed tomography images from Patient 2 after the patient had presented with vomiting

Obstruction of the horizontal portion of the duodenum ( $a, b$, white arrows) and mild abnormal tissue stranding in the retroperitoneum from the pelvis to the duodenum (b, c, black arrowheads) are visible. A thickened bladder wall (d, gray arrowhead) with a malignant target sign in the rectum (d, black arrow) and thickened mesorectal fascia (d, white arrowheads) are also visible. 

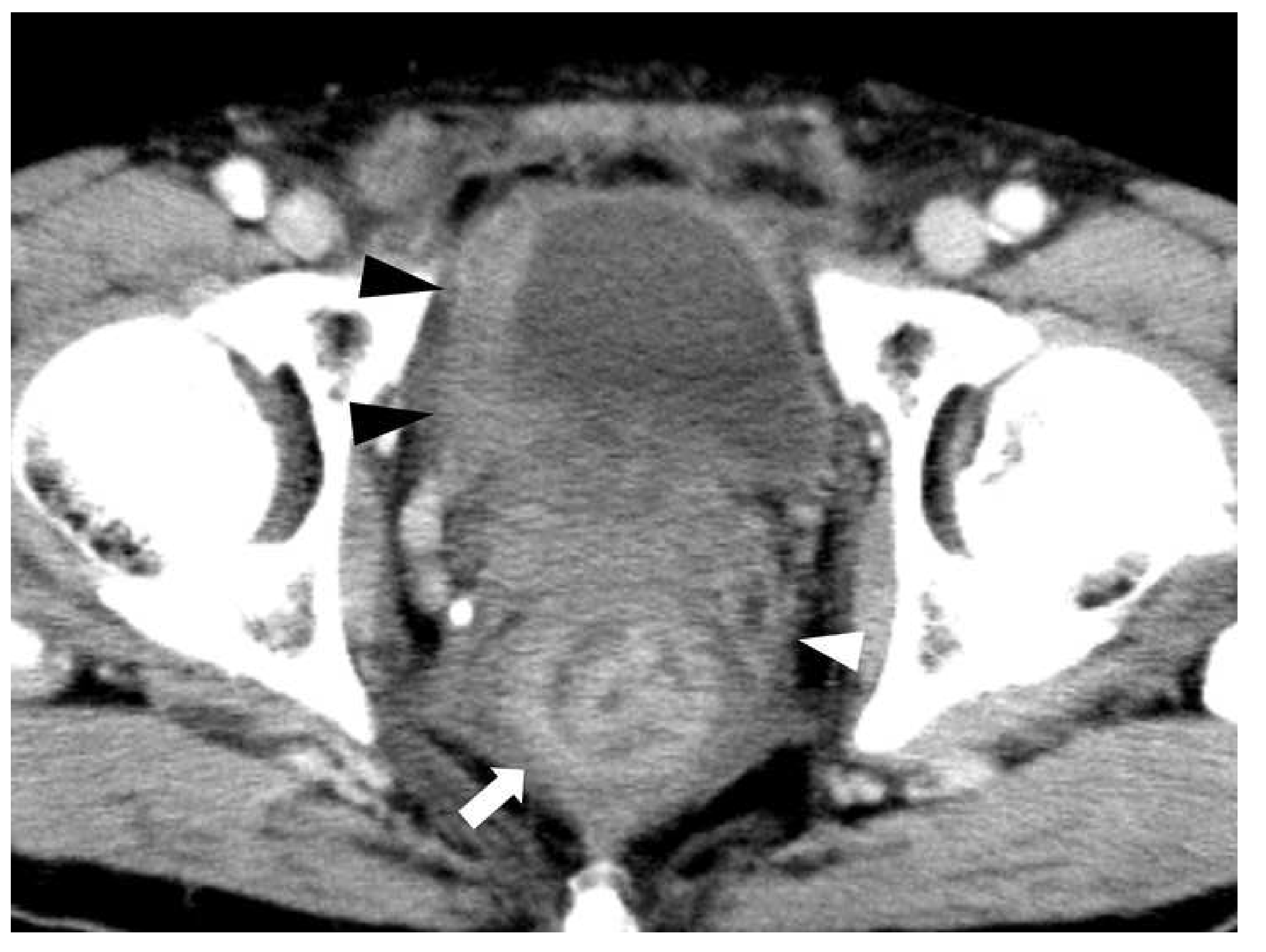
Click here to access/download;Figure;Fig.2.TIF \pm

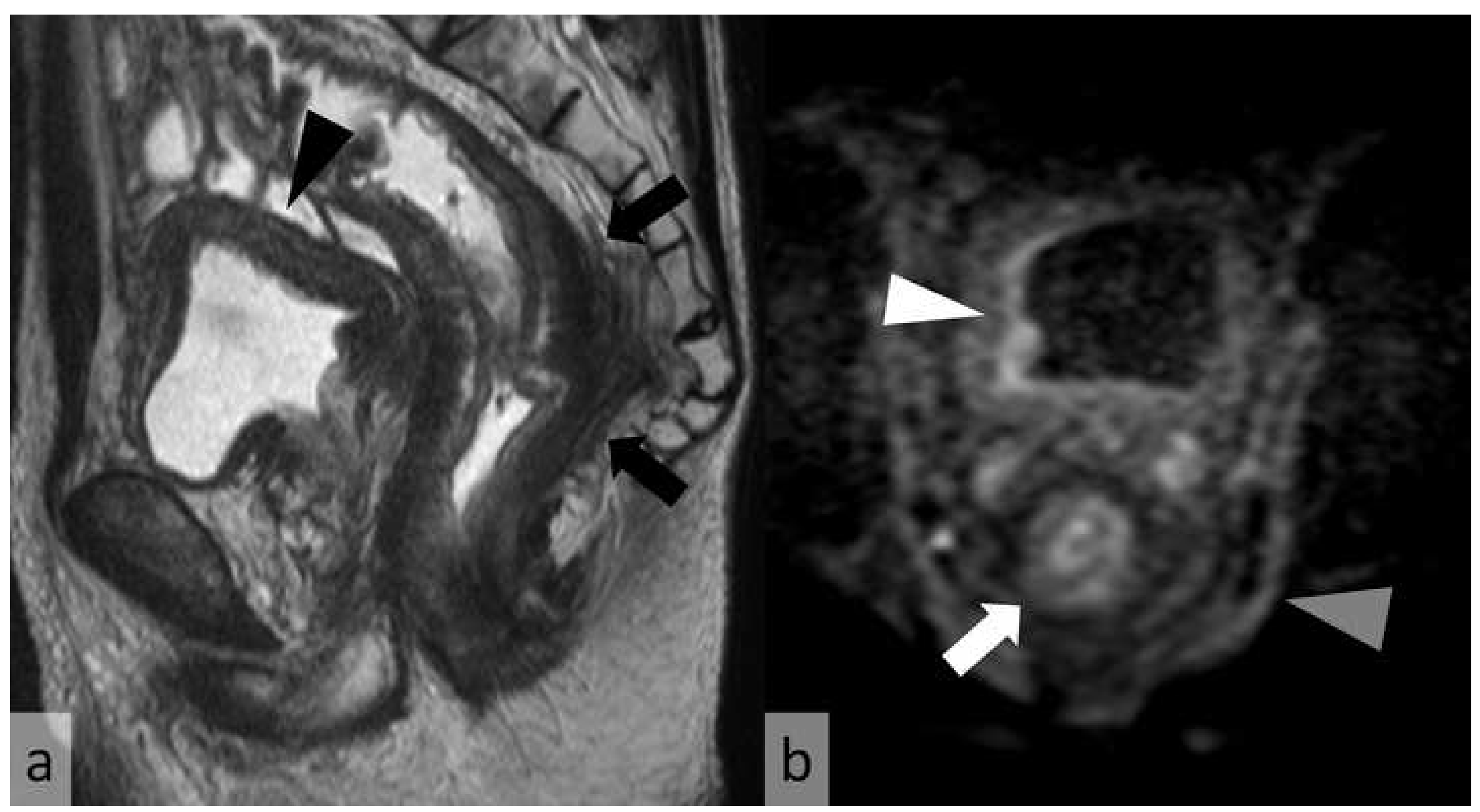



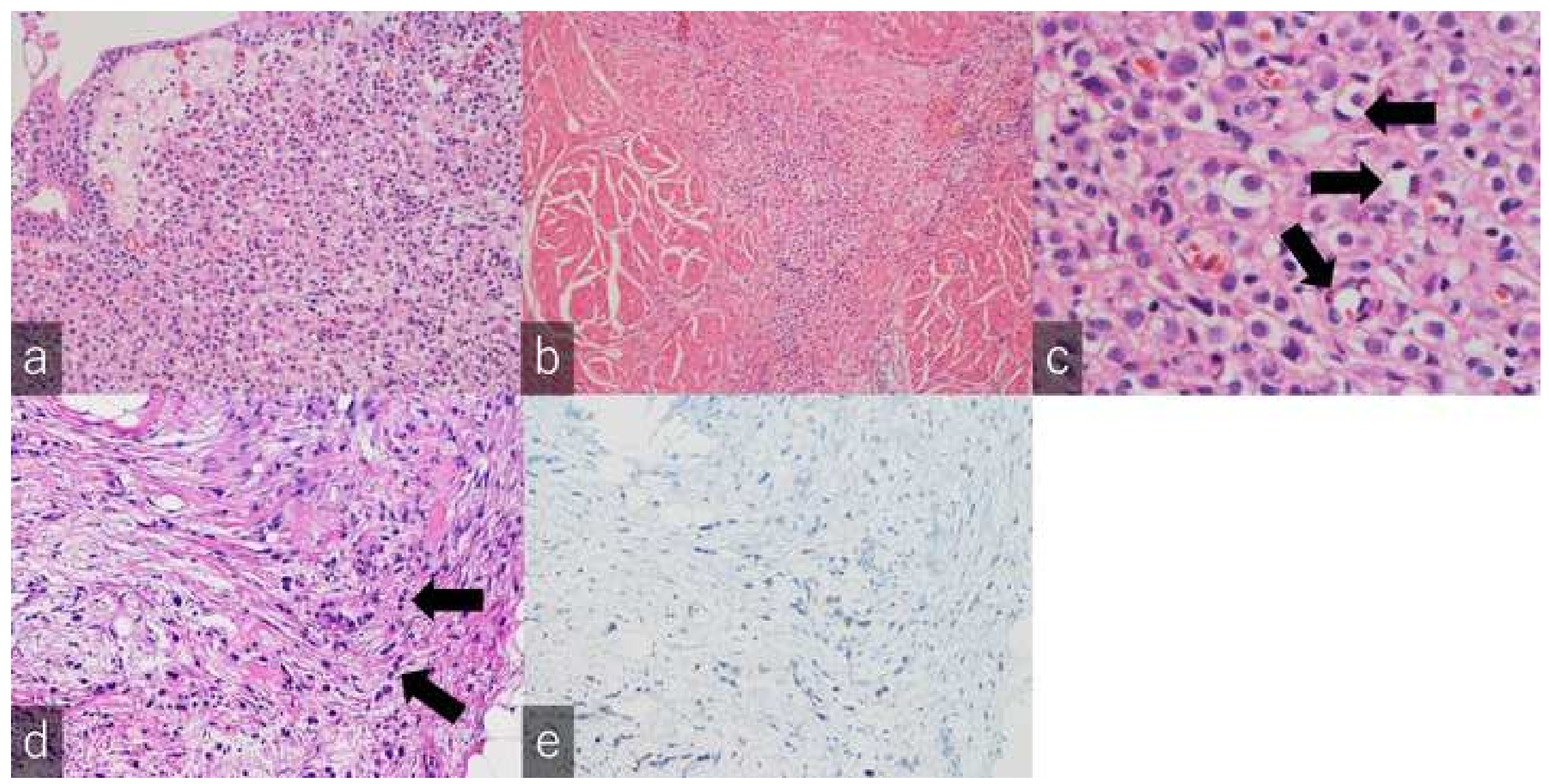


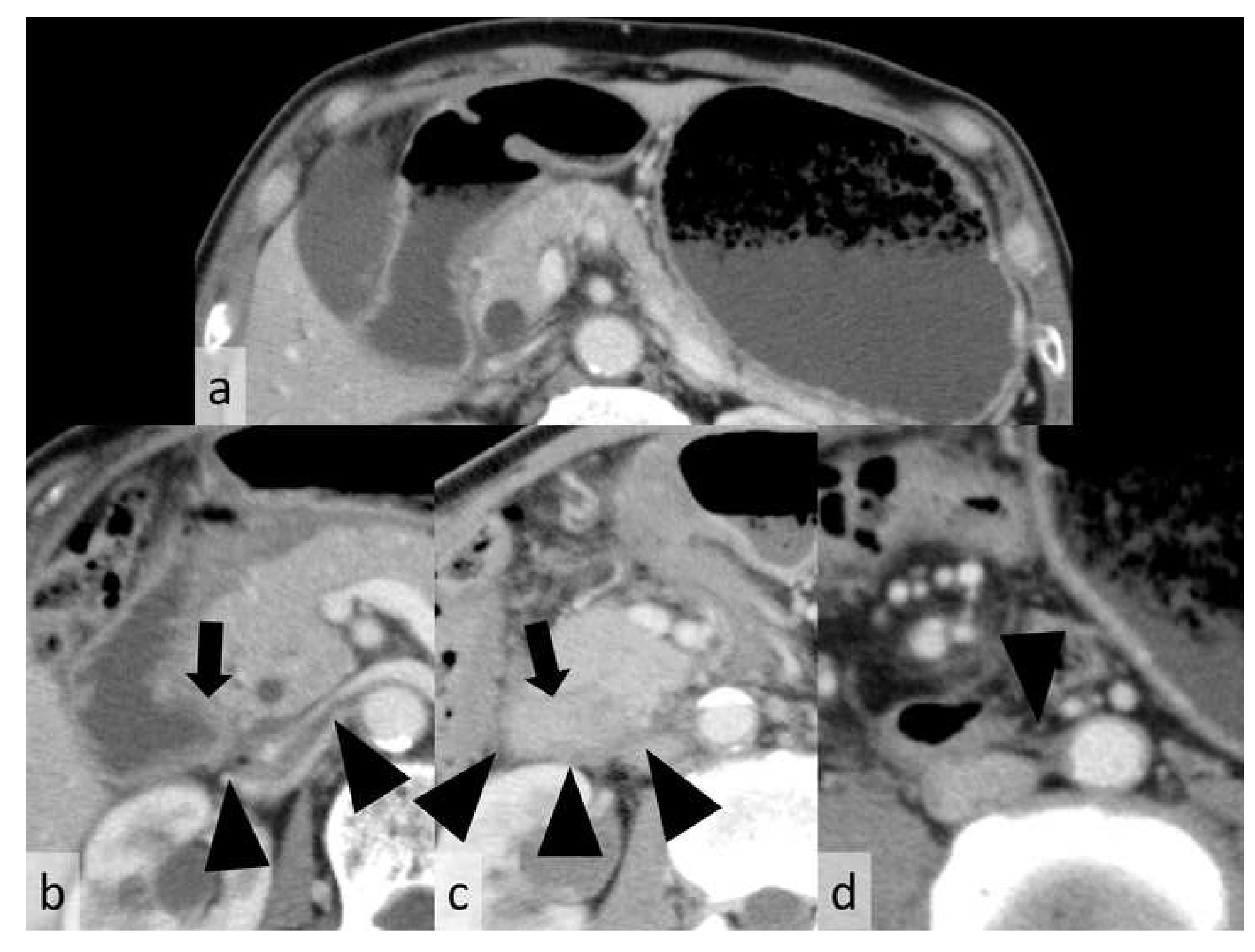









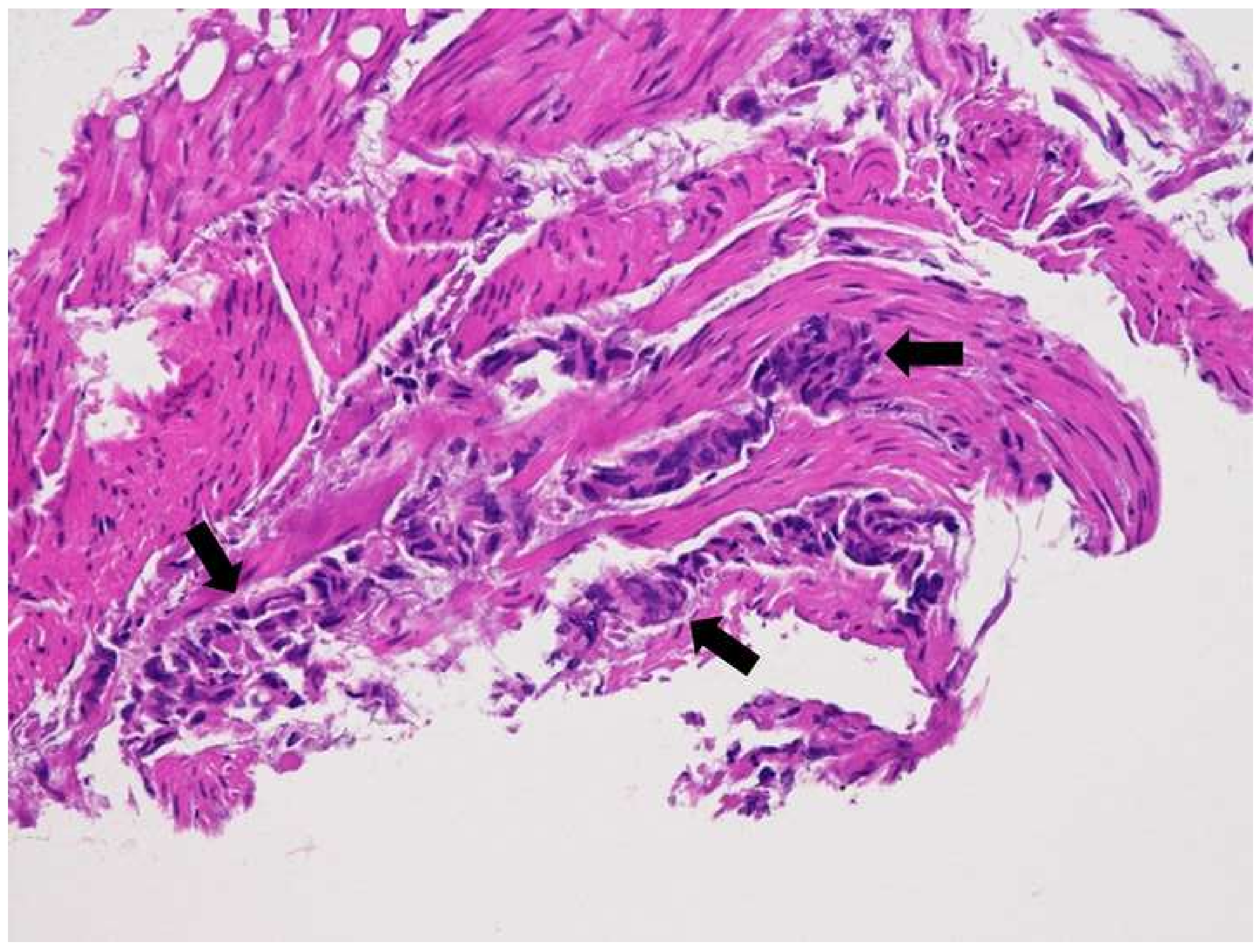




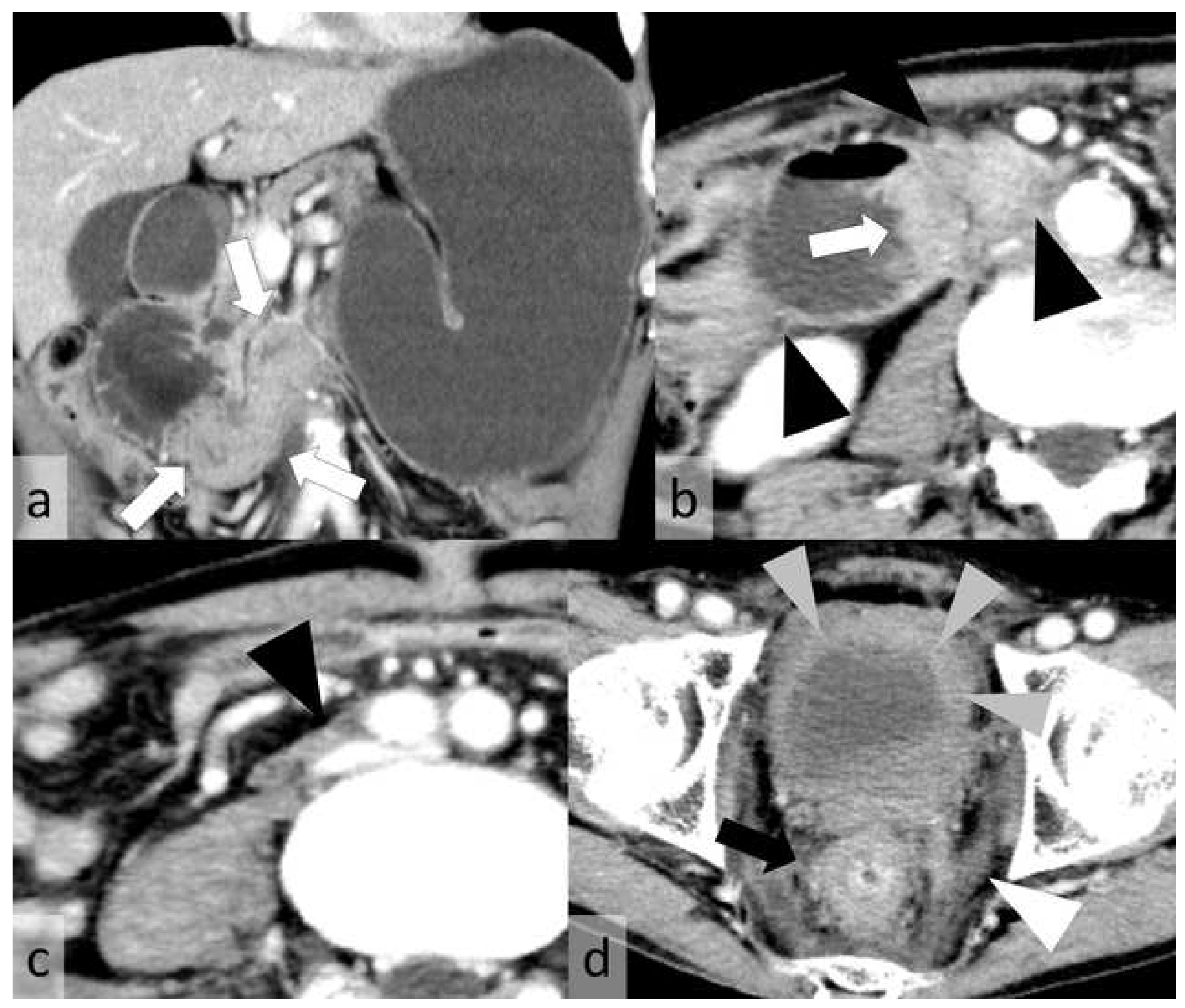

